\title{
Tunable Plasmonic Lattices of Silver Nanocrystals
}

\author{
Andrea Tao ${ }^{1,2}$, Prasert Sinsermsuksakul ${ }^{1}$, and Peidong Yang ${ }^{1,2}$ \\ ${ }^{1}$ Department of Chemistry, University of California, Berkeley \\ Berkeley, CA. 94720 \\ ${ }^{2}$ Materials Sciences Division, Lawrence Berkeley National Laboratory \\ 1 Cyclotron Road, Berkeley, CA. 94720 \\ Email: p_yang@berkeley.edu
}

\begin{abstract}
Silver nanocrystals are ideal building blocks for plasmonic materials that exhibit a wide range of unique and potentially useful optical phenomena. Individual nanocrystals display distinct optical scattering spectra and can be assembled into hierarchical structures that couple strongly to external electromagnetic fields. This coupling, which is mediated by surface plasmons, depends on their shape and arrangement. Here we demonstrate the bottom-up assembly of polyhedral silver nanocrystals into macroscopic two-dimensional superlattices using the Langmuir-Blodgett technique. Our ability to control interparticle spacing, density, and packing symmetry allows for tunability of the optical response over the entire visible range. This assembly strategy offers a new, practical approach to making novel plasmonic materials for application in spectroscopic sensors, sub-wavelength optics, and integrated devices that utilize field enhancement effects.
\end{abstract}


Plasmonic materials are emerging as key platforms for applications that rely on the manipulation of light at small length scales. Materials that possess sub-wavelength metallic features support surface plasmons - collective excitations of the free electrons in a metal - that can locally amplify incident electromagnetic fields by orders of magnitude at the metal surface. This field enhancement is responsible for the host of extraordinary optical phenomena exhibited by metal nanostructures, such as resonant light scattering, surface-enhanced Raman scattering ${ }^{1,2}$, superlensing ${ }^{3}$, and light transmission through optically thick films ${ }^{4,5}$. The shape, size, and periodicity (for arrays) of the metal features explicitly determine the profile and intensity of the observed optical response ${ }^{5-8}$. However, exploiting the field enhancement offered by plasmonic materials for practical applications is limited by lack of a simple method that can generate these nanostructures with geometric control and regularity.

To address these issues, new lithographic and patterning techniques have been developed to improve upon conventional serial methods of templating nanoscale features from evaporated metal films ${ }^{9,10}$. However, all of these methods are limited in the feature sizes and geometries that can be accessed and offer little control over surface roughness, which has important implications in generating local fields with high radiative efficiencies ${ }^{11}$. An alternative strategy is a bottomup approach, where hierarchical architectures are constructed from individual metallic elements. Colloidal Ag and Au nanocrystals (NCs) can be readily synthesized with controlled shapes, sizes, and surfaces and are ideal building blocks for rationally designed plasmonic materials because they generate plasmon-mediated evanescent fields near their surfaces when irradiated with light. 
NC assemblies are optimal platforms for plasmonic applications because they promote electromagnetic coupling between metallic NCs, leading to narrow plasmon bands ${ }^{12}$ and intense field enhancement in the interstitial spaces between neighboring $\mathrm{NCs}^{13,14}$. However, the rational design of bottom-up plasmonic materials is greatly restricted by the ability to construct welldefined, reproducible structures composed of many NCs. Over the past decade, materials research has focused on self-assembly ${ }^{15}$, hard- and soft-templating ${ }^{16,17}$, and evaporation-based ${ }^{18}$ approaches to overcome this challenge of assembly at the nanoscale. Molecular assembly strategies for metal nanoparticles such as the use of DNA-linkages ${ }^{19,20}$, selectively binding ligands $^{21}$, or molecular scaffolding ${ }^{22}$ have been shown to generate complexes of nanoparticles, but with ordering only for small groupings. For application in surface-enhanced Raman sensing or for device integration, large-scale nanoparticle assemblies are needed. Colloidal films generated for these purposes composed of randomly oriented nanoparticle structures exhibit collective electromagnetic effects, but with low regularity and without capability of optimizing the observed response ${ }^{23-25}$. Langmuir-Blodgett (LB) assembly is a robust technique used to form ordered nanoparticle domains over areas of several square centimeters, which can then be easily transferred to a solid substrate. The lack of photolithography or serial writing in this fabrication process makes LB assembly a reliable bottom-up method for generating arrays of metallic features smaller than $100 \mathrm{~nm}$.

\section{Shape-Controlled Nanocrystal Building Blocks}

Here we demonstrate the bottom-up fabrication of large-area, two-dimensional (2D) plasmonic materials composed of polyhedral Ag NCs, where plasmon resonances can be explicitly tuned by NC shape and arrangement. In the LB technique, a colloidal solution of NCs is spread at an air- 
water interface, forming an isotropic monolayer of floating NCs whose density can be controlled via surface pressure (П) through isothermal compression or expansion. Close-packed arrays of NCs can be easily obtained by compressing the fluid-supported film to maximum surface pressures of $\Pi \sim 14 \mathrm{mN} / \mathrm{m}$. Figure 1 shows scanning electron microscopy (SEM) images of superlattices composed of truncated cubes ( $d \sim 120 \mathrm{~nm})$, cuboctahedra ( $d \sim 150 \mathrm{~nm})$, and octahedra ( $d \sim 250 \mathrm{~nm})$ Ag NCs. The different polyhedral shapes are synthesized by a modified polyol process, where the faceted NCs are stabilized by the long-chain polymer, poly(vinyl pyrrolidone) (PVP) $\left(\mathrm{M}_{\mathrm{w}} \sim 55,000\right)^{26}$. Lattice packing and dimensions are dictated explicitly by the choice of NC building blocks: truncated cubes tend to assemble face-to-face to form a square lattice, similar to packing architectures observed for iron particles ${ }^{27}$ and zeolite arrays ${ }^{28}$ (Fig. 1a); for cuboctahedra, a more truncated polyhedral building block, this square lattice shears to form a rhombohedral unit cell (Fig. 1b); octahedra adopt a hexagonal lattice upon compression, laying flat on their triangular facets such that three NCs form an interlocked triangle (Fig. 1c). Correspondingly, when considering the complementary geometries of the interstitial spaces within the lattice, each NC building block also gives rise to unique interstitial structures. Optical transmission and reflectance measurements indicate that these three different symmetries of NC packing profoundly affect the manner in which light interacts with the NC films. The distinct surface plasmon modes of the differently shaped building blocks and the geometries in which they are assembled contributed to collective plasmonic properties that are unique to each LB film (See Supporting Info, S1).

\section{Plasmonic Response of Nanocrystal Assemblies}

To understand the nature of plasmon coupling within these superlattices and explore tunability of 
the resulting optical response, we focused on characterizing the optical properties of cuboctahedra NC monolayers. In colloidal solution, cuboctahedra NCs exhibit a unique electromagnetic response due to their non-spherical geometries, displaying sharp plasmon resonances in the visible regime corresponding to charge polarizations localized at the atomically-defined corners, edges, and facets of the particles ${ }^{26}$. Within a 2D lattice, however, plasmon coupling between NCs is expected to dominate the optical response with increased electromagnetic coupling between NCs as interparticle distance is decreased. This relationship allows for a simple strategy to tailor the optical response of our superlattice structures during the assembly process, specifically by controlling surface pressure at the air-water interface to give different NC spacings. The pressure-area isotherm for a typical compression experiment is shown in Fig. 2a, where changes in slope correspond to phase transitions of the NC film from gas to condensed-liquid to solid states. Snapshots of the cuboctahedra monolayer as it is compressed are shown in Fig. 2(b-d), demonstrating the visually arresting color evolution of the film stemming from changes in the plasmon response due to electromagnetic coupling between NCs. The intensity of the monolayer color at these varying surface pressures is quite surprising given that the film is only a single NC thick; colloidal solutions of the cuboctahedra NCs at much higher concentrations exhibit light reflectance with intensities orders of magnitude lower.

The collective plasmon properties of the NC film are entirely dependent on NC arrangement. To characterize this, we collected samples of the NC monolayer by dip-transfer onto solid supports at low $(\Pi \sim 0 \mathrm{mN} / \mathrm{m})$, intermediate $(\Pi=1 \mathrm{mN} / \mathrm{m})$, and high surface pressures $(\Pi=14 \mathrm{mN} / \mathrm{m})$ corresponding to different phases of the LB film. Scanning electron microscope (SEM) images of these films are shown in Fig. 2(b-d). For $\Pi \sim 0 \mathrm{mN} / \mathrm{m}$, the SEM image in Fig. 2b shows that the 
NCs self-organize into a hexagonal lattice with an interparticle spacing of $\sim 40 \mathrm{~nm}$ and a lattice spacing of $a \sim 230 \mathrm{~nm}$. The distance between NCs is approximately the radius of gyration for two PVP layers ${ }^{29}$, which is expected for as-synthesized Ag NCs that possess surfaces capped by adsorbed polymer. The steric nature of PVP allows for hard-sphere-like interactions between adjacent NCs and the NCs pack into a highly uniform 2D lattice. The SEM image of the NC film obtained at $\Pi=1.0 \mathrm{mN} / \mathrm{m}$ (Fig. 2c) shows that the monolayer is comprised of oligomers between 1-10 NCs large and on average 4 NCs wide. At this threshold surface pressure, the compression force is high enough to overcome the steric barrier of the adsorbed PVP on the NC surfaces that prevented particle aggregation at lower surface pressures. Fig. 2d shows an SEM image of the NC monolayer collected at $\Pi=14 \mathrm{mN} / \mathrm{m}$. Here, NC density reaches a maximum with the distance between neighboring NCs $<5 \mathrm{~nm}$ and estimated to be $\sim 2 \mathrm{~nm}$ when considering the chain length of adsorbed PVP on the Ag surface.

A unique feature of these NC monolayers is the ability to tune the plasmon response of the fluidsupported film across the visible range by controlling electromagnetic interaction. The nature of LB assembly offers the unprecedented opportunity to examine the evolution of NC film reflectance as interparticle spacing is continuously tuned. This is already clearly observed from the digital images shown in Fig 2(b-d). Figures 3(a, b) show the specular reflectance of the cuboctahedra superlattice at the air-water interface as it undergoes isothermal compression, with spectra grouped into low $(\Pi<0.5 \mathrm{mN} / \mathrm{m})$ and high $(0.5<\Pi<14 \mathrm{mN} / \mathrm{m})$ surface pressure regimes for clarity. The reflectance lineshape of the fluid-supported monolayer differs greatly from that of the isotropic colloidal NC suspension, which exhibits a broad dipolar resonance at $\lambda_{\text {dip }} \sim 790$ $\mathrm{nm}$ and a quadrupolar resonance at $\lambda_{\text {quad }} \sim 480 \mathrm{~nm}$ (Fig. 3a inset). At low pressures, a single 
plasmon resonance peak associated with quadrupolar mode coupling dominates the optical response of the water supported NC film (Fig. 3a, blue shaded region). As the film is compressed, this peak blueshifts and increases in reflectance intensity, indicating stronger nearfield interactions between NCs as expected. This peak reaches a maximum intensity just as the film transitions from gas to liquid (corresponding to point b in Figure 2), with a FWHM=48 nm at $\lambda \sim 490 \mathrm{~nm}$. As surface pressure increases with further compression of the monolayer, higherorder mode coupling gives rise to reflectance peaks at shorter wavelengths (Fig. 3a and 3b, yellow-shaded region). In addition, a new broad reflectance peak corresponding to plasmon delocalization over multiple NCs arises in the longer wavelengths (Fig. 3b, green-shaded region). When NC density approaches the close-packed limit at $\Pi=14 \mathrm{mN} / \mathrm{m}$ (point $\mathrm{d}$ in Figure 2), the reflectance spectrum approaches a continuum with uniform intensity rather than exhibiting distinct resonance modes. Clearly, these changes in wavelength and intensity of the film's plasmonic response indicate complex near-field interactions which are dictated by more than a simple distance dependence.

\section{Low-Density Nanocrystal Assemblies}

For $\Pi \sim 0 \mathrm{mN} / \mathrm{m}$, in-plane periodicity gives rise to diffractive coupling of surface plasmons and, similar to light localization for a photonic crystal, the sharp resonance observed in the reflectance spectra is only observed at wavelengths where the conditions for Bragg diffraction (i.e., $\lambda=2 a \sin \theta$, where $\theta=90^{\circ}$ for backscattering collection) are met. This dependence gives a facile method for optimizing the plasmonic response of the film at a given wavelength, since controlling surface pressure will control the lattice constant $a$ for the NC array. This diffractive coupling is evidenced in Figure 3a, where the coupled plasmon mode (blue-shaded) blueshifts as 
the monolayer is compressed and total film area is decreased. Thus, for NC monolayer in the low surface pressure regime, two factors dictate optical properties: (1) the strength of local electromagnetic coupling between NCs and (2) the diffraction conditions dictated by the longrange order of the NC film.

To demonstrate this tunability at low surface pressures, we have optimized the coupled plasmon response for NC superlattices composed of truncated cubes and octahedra using LB assembly (See Supporting Info, S2). For films with surface pressures of $\Pi \sim 0 \mathrm{mN} / \mathrm{m}$, the NC monolayers all adopt an ordered hexagonal superlattice with particle separation distances $>10 \mathrm{~nm}$. Comparison between the optical response of the NC monolayers and their respective colloidal solutions clearly indicate electromagnetic coupling within the superlattices. Films composed of truncated cubes and octahedra display reflectance peaks at $\lambda \sim 480 \mathrm{~nm}$ and $\lambda \sim 710 \mathrm{~nm}$, respectively, corresponding to the coupled quadrupolar modes of the ordered films. In addition, for films composed of truncated cubes, high reflectance intensity observed in the near-IR wavelengths is attributed to a coupled dipolar mode. Despite their similar packing arrangement and interparticle spacing, each NC film exhibits a distinct optical response due to the shapedependent plasmonic properties and size-dependent lattice constants for each different Ag building block. Plasmon tuning at low surface pressures is easily achieved by tailoring the coherent near-field interactions of the ordered NC array.

\section{Close-Packed Nanocrystal Assemblies}

In the high surface pressure regime, the specular reflectance of the cuboctahedra NC monolayer becomes increasingly complex as further monolayer compression leads to particle aggregation 
(Fig. 3b). Because the NCs nucleate into islands, strong plasmon coupling between adjacent NCs gives rise to local areas of intense electromagnetic interaction rather than coherent coupling across the entire monolayer ${ }^{30}$. This is evidenced in the darkfield scattering spectrum (Fig. 3c), which shows a decrease in intensity for the coupled mode in addition to new peaks at longer wavelengths that are associated with the collective charge polarizations of the aggregate islands. $\mathrm{NC}$ ordering and spacing clearly has important bearing on the collective electromagnetic interactions of the entire film (Fig. 3c). Corresponding digital images of the LB films under darkfield illumination are shown in Fig. 3(d-f), displaying the dramatic difference in optical response.

To probe the origin of the surface plasmon resonances associated with multiple particles, we investigated the optical response for small linear assemblies of NCs. Figure 4a shows representative scattering data for a single cuboctahedron, a dimer, and a linear tetramer. SEM images (insets) show that the coupled NCs are spaced $<5 \mathrm{~nm}$ apart. Theoretical scattering spectra for each arrangement (Fig. 4b) were obtained using the discrete dipole approximation method, where coupled NCs were modeled as continuous metallic structures (separation distance $=0 \mathrm{~nm}$, electrical contact). For example, a line of four NCs is approximated as a corrugated rod with an aspect ratio of 1:4. Although experimentally the NCs maintain some interparticle separation due to adsorbed polymer, the simulations correspond well with the measured optical spectra; a redshift in the spectra can be attributed to the inhomogeneous dielectric environment of the glass substrate. For light scattering of the dimer, we observe two peaks corresponding to in-phase dipole coupling in the transverse $(\lambda \sim 515 \mathrm{~nm})$ and longitudinal directions $(\lambda \sim 595 \mathrm{~nm})$ of the dimer axis, similar to the optical response observed for one-dimensional nanostructures such as 
rods and wires. For the tetramer, the blueshift of the transverse mode $(\lambda \sim 490 \mathrm{~nm})$ and the redshift of the longitudinal mode $(\lambda \sim 660 \mathrm{~nm})$ are consistent with theoretical predictions for dipole interactions within chain-like assemblies of $\mathrm{N}$ particles ${ }^{30}$. For the tetramer, the scattering spectrum also displays additional scattering peaks at $\lambda \sim 590 \mathrm{~nm}$ and $\lambda \sim 550 \mathrm{~nm}$ which are assigned to out-of-phase dipole coupling within the four-particle chain. These modes are not observed for rods, but instead are unique to assemblies of touching nanoparticles where electromagnetic coupling between particles is at the strong limit ${ }^{31}$. This coupling is in part facilitated by the well-defined, flat facets of the Ag cuboctahedra that provide an exceptionally large area for interaction. Thus, for NCs with interparticle distances $<5 \mathrm{~nm}$, intense near-field interactions between NCs allows for surface plasmon modes associated with the entire NC aggregate.

This near-field interaction also has important consequences when considering the close-packed NC monolayer. Upon further compression of the LB monolayer to surface pressures $\Pi>5 \mathrm{mN} / \mathrm{m}$, the NC islands coalesce into a uniform close-packed superlattice. Electromagnetic coupling is evidenced in the scattering spectrum, which approaches a continuum rather than exhibiting distinct resonance peaks. As in the case of the NC aggregates, intense near-field interaction between NCs facilitates charge polarizations associated with multiple NCs; for the close-packed film, however, this collective effect occurs over the entire NC film. Near-field scanning optical microscope (NSOM) images indicate that this long-range electromagnetic interaction enables propagating surface plasmons, similar to those observed for continuous or corrugated silver surfaces, which can be excited at defects in the crystalline array (See Supporting Info, S3). NSOM images indicate low intensity variance for ordered NC domains, unlike films of isolated 
NCs that clearly display localized spots of high and low intensities. For nearly continuous metal films, this response has been attributed to propagating surface waves ${ }^{32}$. As a consequence, the NC monolayer resembles a metallic thin film, displaying a high reflectivity and a characteristic sheen (point d in Fig. 2). Close-packed NC superlattices also exhibit a broad reflectance range similar to a bulk metal surface (See Supporting Info, S1). These observations, along with the demonstrated versatility of the LB approach for organizing a wide range of nanostructures, suggest that propagating surface plasmons can be properly engineered through a bottom-up approach.

We illustrate the potential of a bottom-up strategy for engineering novel optical materials by generating 2D plasmonic superlattices from Ag NC building blocks. The LB technique can readily achieve novel sub-wavelength features and can be implemented over macroscopic areas with relative ease, providing a practical mean of integration into current device architectures. This bottom-up approach also offers a high degree of tunability by exhibiting exceptional control over the nanoscale arrangement of the shape-controlled NCs used in this study. This is also the first example where a bottom-up assembly technique has been demonstrated to tune both the frequency and the strength of the plasmonic response for these NC monolayers. In addition, the ability to control the density and strength of near-field interactions between NCs has important consequences for applications that utilize electromagnetic hot spots. Further work on the effects of lattice symmetry and NC linkage on electromagnetic coupling should provide additional flexibility and control of the optical response. These rationally designed plasmonic materials should have a major impact on the realization of technologies such as biological imaging, nanoscale lithography, low-level Raman detection, and integrated plasmonic/photonic devices. 


\section{Methods}

We synthesized all of the colloidal nanocrystal solutions using a previously reported polyol reaction ${ }^{26}$. The as-made colloidal solutions were washed by centrifugation and redispersed in a 1:1 mixture of ethanol and deionized water. This solution was pulled through three differently sized Millipore DVPP Durapore filter membranes: 650 nm, 450 nm, and 220 nm.

To prepare the nanocrystals for LB assembly, we centrifuged this filtered solution and redispersed the precipitate in small amount of ethanol $(\sim 100 \mu \mathrm{l})$ before diluting with $5-10 \mathrm{~mL}$ $\mathrm{CHCl}_{3}$. To remove any dust particles or organic material, the Teflon-coated LB trough (Nima, Type 611) was wiped with $\mathrm{CHCl}_{3}$ and then purged with deionized water. We added this solution drop-wise to the air-water interface of the LB trough. When the colloidal NC solution is added, the $\mathrm{CHCl}_{3}$ quickly spreads on the water surface and evaporates to leave an isotropically distributed monolayer of NCs floating at the air-water interface. After adding the desired amount of colloidal starting material, the film was allowed to equilibrate for at least 30 minutes. This monolayer is then isothermally compressed by moving the barrier across the interface until a desired surface pressure (П) is achieved. Isothermal compression was performed at a barrier speed of $10 \mathrm{~cm}^{2} / \mathrm{min}$ for surface pressures of $\Pi<5 \mathrm{mN} / \mathrm{m}$. For $\Pi>5 \mathrm{mN} / \mathrm{m}$, compression was performed at a barrier speed of $5 \mathrm{~cm}^{2} / \mathrm{min}$.

In-situ reflectance measurements during compression were performed using a UV-Vis spectrometer (Agilent, UV-Vis Chemstation 8453) equipped with a fiber optic coupler (Custom Sensors and Technology, Agilent Fiber Optic Coupler Model 9001B) and two single-mode fiber 
optic cables for illumination and collection. The two fibers were positioned vertically above the water-filled LB trough at a 15-degree angle of incidence. For blank/background calibration, spectra were also collected for highly reflective solid substrates with known reflectance curves (silicon, silver mirror) using the same experimental setup.

We transferred the NC monolayers off the air-water interface onto solid substrates using a vertical dip-coating method. Glass and silicon substrates were cleaned by sonication in isopropanol. Before compression, the desired substrate was immersed vertically into the water subphase. After compression, the substrates were slowly pulled out of the water at a dipping speed of $1 \mathrm{~mm} / \mathrm{min}$. For high surface pressure films, both hydrophobic and hydrophilic substrates resulted in near perfect monolayer transfer. For low surface pressure films $(\Pi<0.5 \mathrm{mN} / \mathrm{m})$, only hydrophilic surfaces such as glass or $\mathrm{O}_{2}$ plasma-treated silicon substrates resulted in efficient film transfer.

Darkfield scattering measurements were performed with an inverted microscope (Olympus, IX71). Samples were then illuminated by a $100 \mathrm{~W}$ halogen bulb using a darkfield condenser (NA=1.2-1.4) with immersion oil. Light was collected through a 60x microscope objective lens (NA=0.7) and captured by a 1340x400 pixel, back-illuminated CCD (Princeton Instruments, Spec-10:400B) and spectrometer (Princeton Instruments, SpectraPro 2300i).

\section{Acknowledgement:}

This work was supported by the Office of Basic Science, Department of Energy. A.R.T. acknowledges the National Science Foundation for a graduate research fellowship. We thank the National Center for Electron Microscopy for the use of their facilities. 
Figures

Figure 1
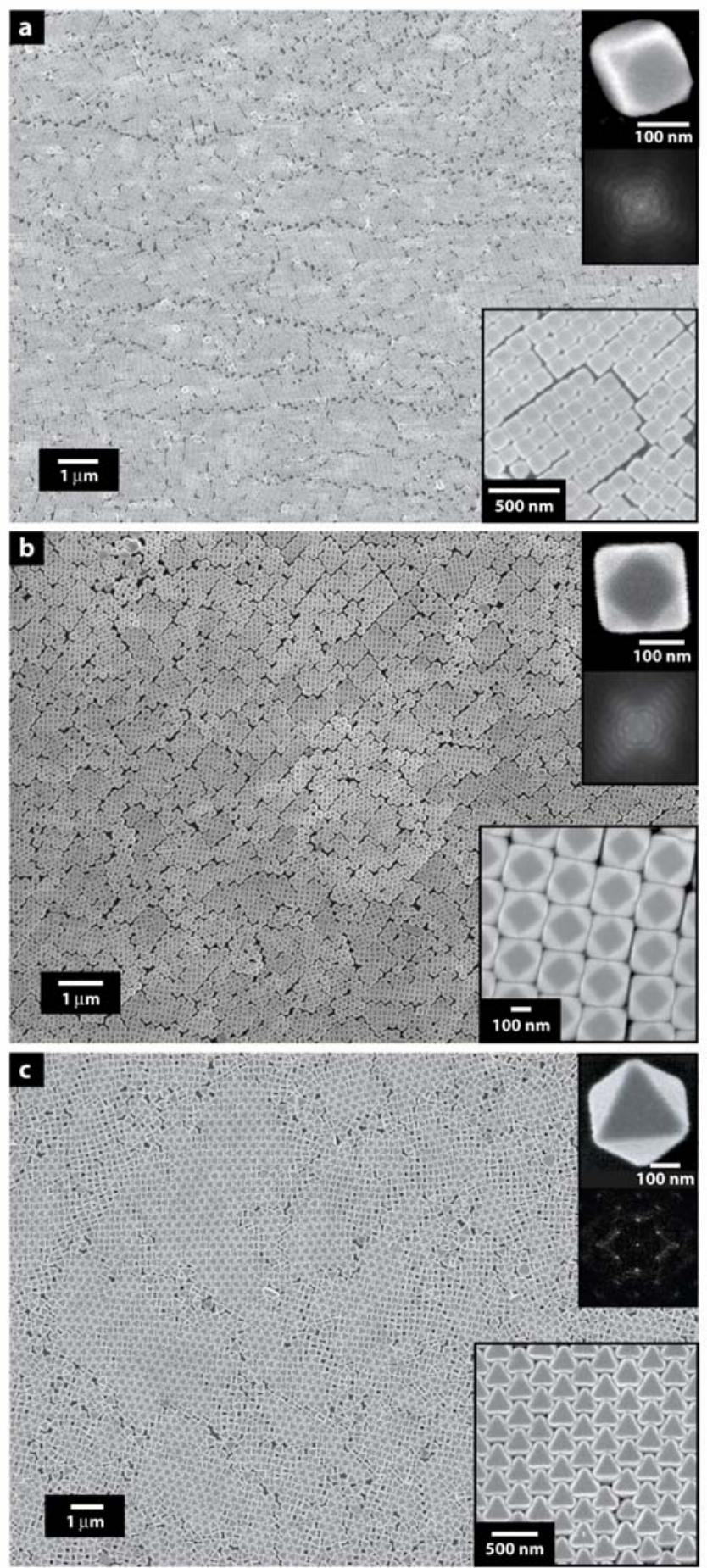

Fig. 1. Novel superlattice architectures comprised of different polyhedral building blocks. Closepacked NC monolayers in these SEM images were obtained after isothermal compression of a LB NC monolayer at a compression rate of $\sim 10 \mathrm{~cm}^{2} / \mathrm{min}$ to a final surface pressure of $\Pi=14$ $\mathrm{mN} / \mathrm{m}$. Superlattices were assembled with (a) truncated cubes, (b) cuboctahedra, and (c) 
octahedra Ag NCs. Insets: individual nanocrystal geometry (top); FFT images indicate longrange order within each monolayer (middle); close-up SEM image showing the nanocrystal unit cell (bottom). 


\section{Figure 2}
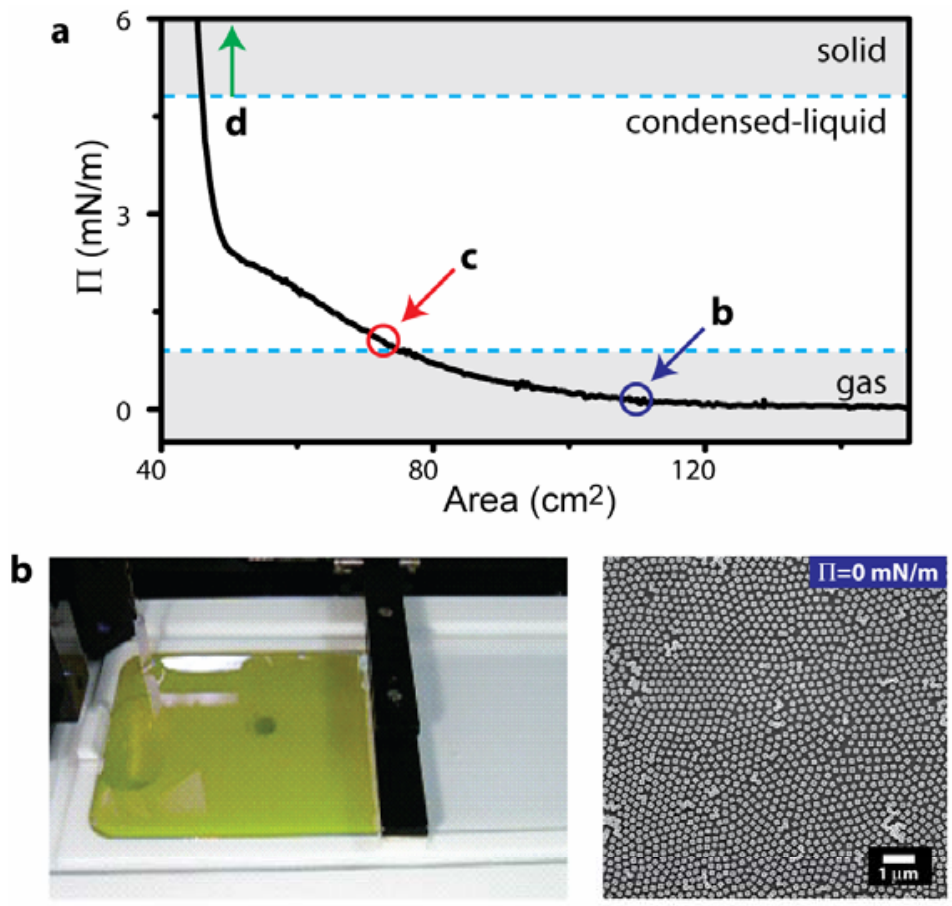

c
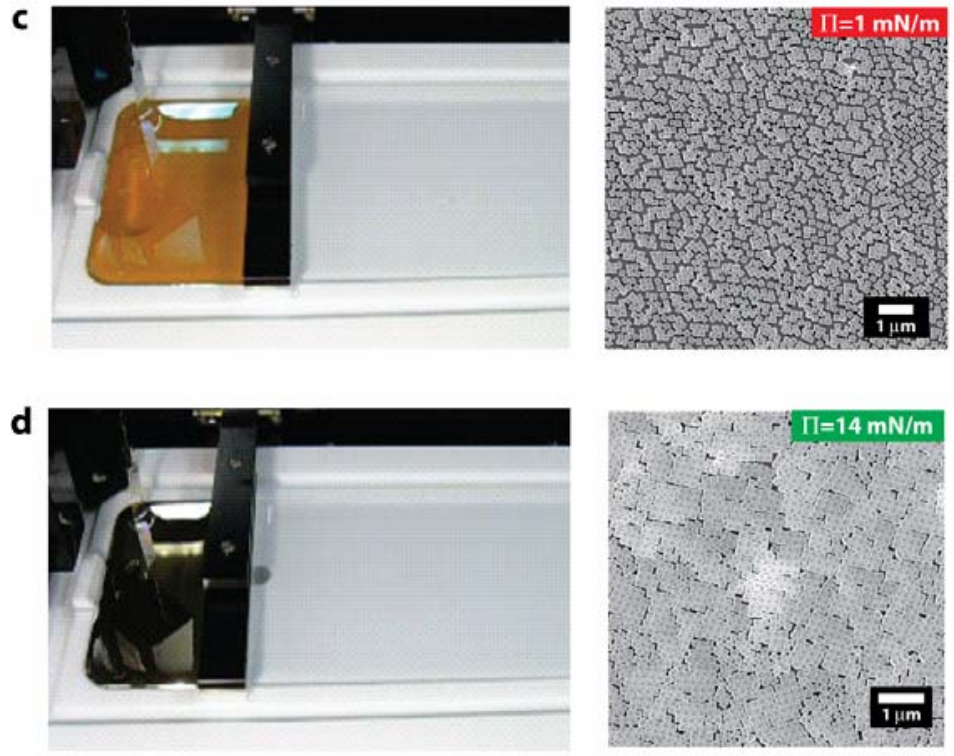

Fig. 2. The tunable plasmon response of a Ag cuboctahedra superlattice as a function of surface pressure. (a) Typical pressure-area isotherm for a Langmuir-Blodgett compression. The NC monolayer undergoes a series of phase transitions as surface pressure is increased, indicated by a change in the isotherm slope. Isothermal compression was performed at a barrier speed of 10 $\mathrm{cm}^{2} / \mathrm{min}$ for surface pressures of $\Pi<5 \mathrm{mN} / \mathrm{m}$. For $\Pi>5 \mathrm{mN} / \mathrm{m}$, compression was performed at a barrier speed of $5 \mathrm{~cm}^{2} / \mathrm{min}$. (b-d) Snapshots of the fluid-supported NC film and corresponding SEM images of film after transfer to a Si substrate during the isothermal compression at surface pressures of (b) $\Pi=0 \mathrm{mN} / \mathrm{m}$, (c) $\Pi=1.0 \mathrm{mN} / \mathrm{m}$, and (d) $\Pi=14 \mathrm{mN} / \mathrm{m}$. As NC density increases via mechanical compression of the film, the evolving coupled plasmon response of the superlattice 
results in vibrant color changes of the bulk NC film. (b) At low NC density ( $\Pi \sim 0 \mathrm{mN} / \mathrm{m})$, the cuboctahedra NCs experience hard-sphere interactions and spontaneously assemble into a hexagonal lattice. (c) As NC density increases $(\Pi=1 \mathrm{mN} / \mathrm{m})$, the cuboctahedra nucleate into small islands composed of 1-10 NCs. (d) At the maximum NC density ( $\Pi=14 \mathrm{mN} / \mathrm{m})$, the Ag NCs adopt a crystalline, close-packed superlattice. The monolayer appears highly reflective and is irreversibly compressed. 


\section{Figure 3}
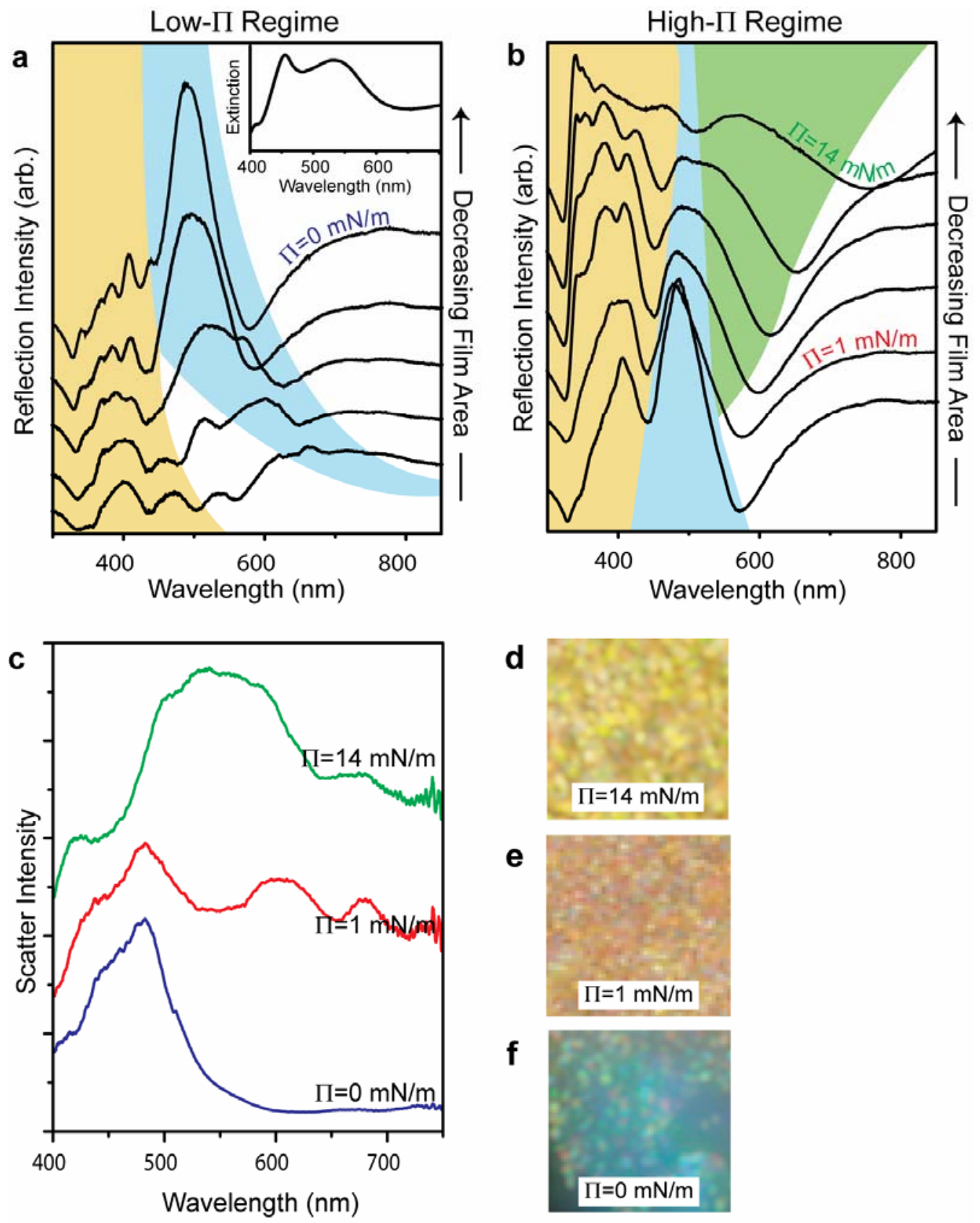

d

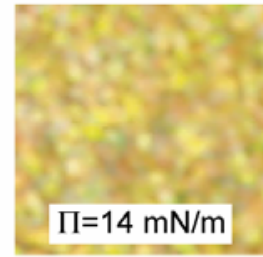

e

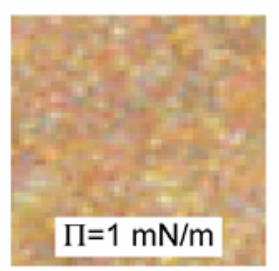

f

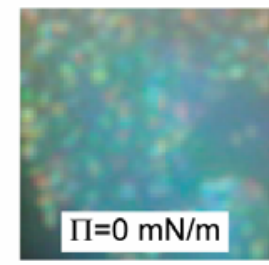

Fig. 3. Evolution of the plasmon response for the NC arrays with increasing surface pressures. (a-b) Specular reflectance measurements obtained in situ at the air-water interface. Spectra are offset for clarity. Total film area decreases from bottom to top for each graph. Color backgrounds are used to highlight plasmon responses arising from quadrupolar coupling (blue), higher-order mode coupling (yellow), and plasmon delocalization (green). (a) At low surface pressures of $\Pi \sim 0 \mathrm{mN} / \mathrm{m}$ during the initial stages of monolayer compression. Inset: Extinction spectrum for a colloidal solution of Ag cuboctahedra NCs. (b) At higher surface pressures from 
$\Pi=0.5-14 \mathrm{mN} / \mathrm{m}$. (c) Darkfield scattering spectra for the NC monolayers at the labeled surface pressures. Spectra are normalized and offset for clarity. (d-f) Digital images of each film under darkfield illumination. 
Figure 4
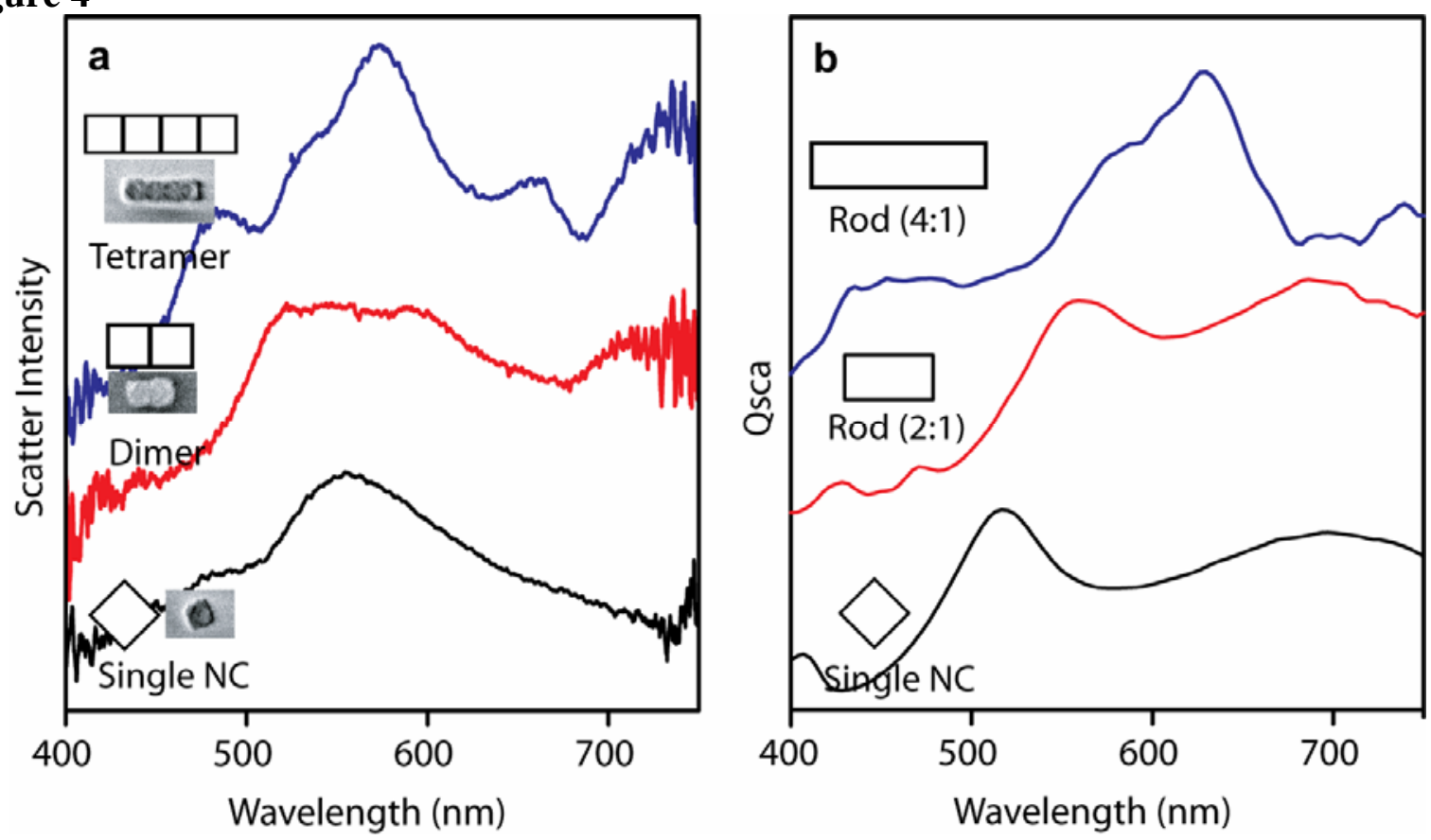

Fig. 4. Optical response of Ag NC oligomers. (a) Darkfield scattering spectra of NC oligomers collected in transmission mode after dispersing a droplet of the colloidal solution onto a glass slide patterned with alignment marks. The number of identifiable plasmon modes increases as oligomer size increases, indicating strong electromagnetic coupling between neighboring cuboctahedra. SEM images show that within the groups, the NCs are in intimate contact with each other (spacing $<5 \mathrm{~nm}$ ). (b) Discrete dipole approximation simulations corresponding to the NC oligomers studied in (A). Cuboctahedra dimers and tetramers are modeled as corrugated rods with aspect ratios of $2: 1$ and $4: 1$, respectively. 


\section{References}

1. Emory, S.R. \& Nie, S. Probing Single Molecules and Single Nanoparticles by SurfaceEnhanced Raman Scattering. Science 275, 1102 - 1106 (1997).

2. Kneipp, K. et al. Single Molecule Detection Using Surface-Enhanced Raman Scattering (SERS). Phys. Rev. Lett. 78, 1667 (1997).

3. $\quad$ Fang, N., Lee, H., Sun, C. \& Zhang, X. Sub-Diffraction-Limited Optical Imaging with a Silver Superlens. Science 308, 534-537 (2005).

4. $\quad$ Ebbesen, T.W., Lezec, H.J., Ghaemi, H.F., Thio, T. \& Wolff, P.A. Extraordinary optical transmission through sub-wavelength hoel arrays. Nature 391, 667-669 (1998).

5. Degiron, A. \& Ebbesen, T.W. The role of localized surface plasmon modes in the enhanced transmission of periodic subwavelength apertures. J. Opt. A 7, S90-S96 (2005).

6. Gao, H., Henzie, J. \& Odom, T.W. Direct Evidence for Surface Plasmon-Mediated Enhanced Light Transmission through Metallic Nanohole Arrays. Nano Lett. 6, 2104 2108 (2006).

7. Molen, K.L.v.d., Segerink, F.B., Hulst, N.F.v. \& Kuipers, L. Influence of hole size on the extraordinary transmission through subwavelength hole arrays. Appl. Phys. Lett. 85, 4316-4318 (2004).

8. $\quad$ Koerkamp, K.J.K., Enoch, S., Segerink, F.B., Hulst, N.F.v. \& Kuipers, L. Strong Influence of Hole Shape on Extraordinary Transmission through Periodic Arrays of Subwavelength Holes. Phys. Rev. Lett. 92, 183901-183904 (2004).

9. Kwak, E.-S. et al. Surface Plasmon Standing Waves in Large-Area Subwavelength Hole Arrays. Nano Lett. 5, 1963-1967 (2005).

10. Fischer, U.C. \& Zingsheim, H.P. Submicroscopic pattern replication with visible light. $J$. Vac. Sci. Technol. 19, 881 (1981).

11. Li, C., Kattawar, G.W. \& Yang, P. Effects of surface roughness on light scattering by small particles. J. Quant. Spectrosc. Ra. 89, 123.

12. Hicks, E.M. et al. Controlling Plasmon Line Shapes through Diffractive Coupling in Linear Arrays of Cylindrical Nanoparticles Fabricated by Electron Beam Lithography. Nano Lett. 5, 1065 -1070 (2005).

13. Garcia-Vidal, F.J. \& Pendry, J.B. Collective theory for surface enhanced Raman scattering. Phys. Rev. Lett. 77, 1163-1166 (1996).

14. Genov, D.A., Sarychev, A.K., Shalaev, V.M. \& Wei, A. Resonant Field Enhancements from Metal Nanoparticle Arrays. Nano Lett. 4, 153-158 (2004).

15. Kalsin, A.M. et al. Electrostatic Self-Assembly of Binary Nanoparticle Crystals with a Diamond-Like Lattice. Science 312, 420-424 (2006).

16. Xia, Y. \& Whitesides, G.M. Soft Lithography. Annu. Rev. Mater. Sci. 28, 153-184 (1998).

17. Manoharan, V.N., Elsesser, M.T. \& Pine, D.J. Dense Packing and Symmetry in Small Clusters of Microspheres. Science 301, 483-487 (2003).

18. Narayanan, S. \& Wang, J. Dynamical Self-Assembly of Nanocrystal Superlattices during Colloidal Droplet Evaporation by in situ Small Angle X-Ray Scattering. Phys. Rev. Lett. 93, 135501-135504 (2004).

19. Mirkin, C.A., Letsinger, R.L., Mucic, R.C. \& Storhoff, J.J. A DNA-based method for rationally assembling nanoparticles into macroscopic materials. Nature 382, 607 - 609 (1996). 
20. Sonnichsen, C., Reinhard, B.M., Liphardt, J. \& Alivisatos, A.P. A molecular ruler based on plasmon coupling of single gold and silver nanoparticles. Nat. Biotech. 23, 741 (2005).

21. Andrea Schroedter, H.W. Ligand Design and Bioconjugation of Colloidal Gold Nanoparticles. Angew. Chem. Int. Edit. 41, 3218-3221 (2002).

22. Warner, M.G. \& Hutchison, J.E. Linear assemblies of nanoparticles electrostatically organized on DNA scaffolds. Nat. Mater. 2, 272 (2003).

23. Freeman, R.G. et al. Self-Assembled Metal Colloid Monolayers: An Approach to SERS Substrates. Science 267, 1629-1632 (1995).

24. Maxwell, D.J., Emory, S.R. \& Nie, S. Nanostructued Thin-Film Materials with SurfaceEnhanced Optical Properties. Chem. Mater. 13, 1082-1088 (2001).

25. Musick, M.D., Keating, C.D., Keefe, M.H. \& Natan, M.J. Stepwise Construction of Conductive Au Colloid Multilayers from Solution. Chem. Mater. 9, 1499-1501 (1997).

26. Tao, A.R., Sinsermsuksakul, P. \& Yang, P. Polyhedral Silver Nanocrystals with Distinct Scattering Signatures. Angew. Chem. Int. Edit. 45, 4597-4601 (2006).

27. Dumestre, F., Chaudret, B., Amiens, C., Renaud, P. \& Fejes, P. Superlattices of iron nanocubes synthesized from Fe[N(SiMe3)2]2. Science 303, 821-823 (2004).

28. Lee, J.S.L., Yun-Jo; Tae, Eunju Lee; Park, Yong Soo; Yoon, Kyung Byung Synthesis of Zeolite As Ordered Multicrystal Arrays. Science 301, 818-821 (2003).

29. Sun, T. \& H. E. King, J. Aggregation Behavior in the SemidilutePoly(N-vinyl-2pyrrolidone)/Water System. Macromolecules 29, 3175-3181 (1996).

30. Quinten, M. \& Kreibig, U. Optical properties of aggregates of small metal particles. Surf. Sci. 172, 557 (1986).

31. Romero, I., Aizpurua, J., Bryant, G.W. \& Abajo, F.J.G.d. Plasmons in nearly touching metallic nanoparticles: singular response in the limit of touching dimers. Opt. Express 14, 9988-9999 (2006).

32. Seal, K. et al. Near-field Intensity Correlations in Semicontinuous Metal-Dielectric Films. Phys. Rev. Lett. 94, 226101 (2005). 